Check for updates

Cite this: RSC Adv., 2017, 7, 29959

\title{
Low thermal conductivity in Si/Ge hetero-twinned superlattices $\uparrow$
}

\author{
Huicong Dong, ${ }^{\text {a Bin Wen, (D) *a Yuwen Zhang }}{ }^{\mathrm{b}}$ and Roderick Melnik ${ }^{\mathrm{c}}$ \\ The thermophysical properties of $\mathrm{Si} / \mathrm{Ge}$ hetero-twinned superlattices (SLs) are investigated by \\ nonequilibrium molecular dynamic simulations. The results indicate that the Si/Ge hetero-twinned SLs \\ show low thermal conductivity, which is similar to that of conventional Si/Ge SLs. Analysis with phonon \\ kinetic theory shows that low thermal conductivity in the Si/Ge hetero-twinned SLs is caused by the \\ combined actions of reduced phonon group velocity and reduced relaxation time. Moreover, despite \\ similar thermal conductivity in Si/Ge hetero-twinned SLs and conventional Si/Ge SLs, the phonon group \\ velocity and relaxation time in the two structures are totally different. Our results demonstrate the \\ potential of a new kind of SLs with strong phonon scattering.
}

Received 4th April 2017

Accepted 6th June 2017

DOI: 10.1039/c7ra03833j

rsc.li/rsc-advances

By combining the structure properties of heterostructure

\section{Introduction}

Superlattices (SLS), consisting of periodically alternating nanolayers, are of great interest due to their wide applications in many fields, such as in developing high-performance thermoelectric devices, thermal management in semiconductor lasers, ${ }^{1-5}$ etc. The previous simulated and experimental results indicated that the thermal transport properties in SLs can be adjusted by regulating the lattice period ${ }^{6,7}$ and the structures of interfaces. ${ }^{\mathbf{8}, 9}$

According to the structures of the interfaces, SLs can be divided into two kinds: (1) heterostructure SLs (SLs consist of two components ${ }^{10}$ ), and (2) homo-coherent twinned SLs (SLS consist of nanotwinned layers from one component ${ }^{9}$ ). It has been demonstrated that the cross-plane thermal conductivity of crystalline heterostructure SLs is much lower than that of bulk materials with a single component, and, sometimes even lower than those of their corresponding alloys. ${ }^{11-13}$ The significant reduction of thermal conductivity in heterostructure SLs is caused by numerous strong scatterings at the heterogeneous interfaces. ${ }^{14}$ As for the homo-coherent twinned SLs, the results are rather different that their thermal conductivity is only slightly reduced when compared with that of the corresponding single bulk crystalline, because homo-coherent twin interfaces show weak effect on thermal transmission. ${ }^{9,15}$

\footnotetext{
${ }^{a}$ State Key Laboratory of Metastable Materials Science and Technology, Yanshan University, Qinhuangdao 066004, China.E-mail: wenbin@ysu.edu.cn

${ }^{b}$ Department of Mechanical and Aerospace Engineering, University of MissouriColumbia, Columbia, Missouri, USA

'The MS2Discovery Interdisciplinary Research Institute, Wilfrid Laurier University, 75 University Ave. West, Waterloo, Ontario, Canada N2L 3C5

$\dagger$ Electronic supplementary information (ESI) available. See DOI: $10.1039 / \mathrm{c} 7 \mathrm{ra} 03833 \mathrm{j}$
} nd homo-coherent twinned SLs, a new type of SLs, heterotwinned SLs, with hetero-coherent twin interfaces periodically arranged in, has been proposed and observed in experimentally, which has been reported can be deposited via DC magnetron sputtering at room temperature. ${ }^{16,17}$ Although the mechanisms responsible for mechanical properties of the hetero-twinned SLs have been studied ${ }^{18}$ their thermal properties are still puzzled. To clear this issue, $\mathrm{Si} / \mathrm{Ge}$ hetero-twinned SLs are chosen as the object of study, and their thermal properties have been investigated. The results indicate that $\mathrm{Si} / \mathrm{Ge}$ heterotwinned SLs show low thermal conductivity due to the reduced average phonon group velocity and relaxation time.

\section{Simulation section}

To build Si/Ge hetero-twinned SLs, the $X, Y, Z$ axes of Si layers correspond to the lattice orientations of [110], [1112], [111], and $[\overline{1} 10],[11 \overline{2}],[111]$ for Ge layers. The lattice constant of single crystal $\mathrm{Si}\left(a_{\mathrm{Si}}\right)$ at $0 \mathrm{~K}$ is $5.43 \AA$, and that of single crystal Ge $\left(a_{\mathrm{Ge}}\right)$ is $5.66 \AA$; thus, the lattice misfit between $\mathrm{Si}$ and Ge single crystal is $4 \%$. To build Si/Ge hetero-twinned superlattice samples, an average lattice constant of $\mathrm{Si}$ and $\mathrm{Ge}$, [111], is used to arrange atoms. Fig. 1 shows a typical model of $\mathrm{Si} / \mathrm{Ge}$ hetero-twinned SLs, which is created by periodically stacking $\mathrm{Si} / \mathrm{Ge}$ thin slices on upon the other along the [111] direction. The unit cell (the periodic thickness $D$ is $1.93 \mathrm{~nm}$ ) for the periodic slice of Si/Ge hetero-twinned SLs has been placed in Fig. 1(a), as well as that of conventional Si/Ge SLs in Fig. 1(b), $\mathrm{Si}_{0.5} \mathrm{Ge}_{0.5}$ alloys in Fig. 1(c), single crystal Si in Fig. 1(d), and twinned Si in Fig. 1(e). In simulation, the periodic thickness $D$ of $\mathrm{Si} / \mathrm{Ge}$ hetero-twinned SLs, conventional Si/Ge SLs and twinned Si varies from 1.93 to $57.83 \mathrm{~nm}$. The cross sections of the five models are all $2.05 \mathrm{~nm} \times 1.97 \mathrm{~nm}$, and model lengths along [111] direction range from 81 to $230 \mathrm{~nm}$. 


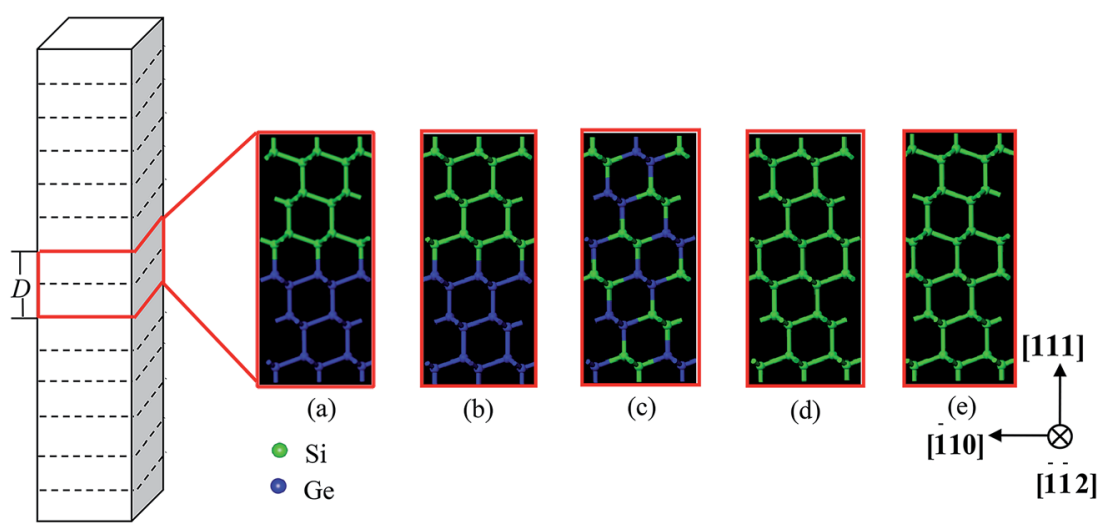

Fig. 1 A schematic diagram for typical [111] oriented structure, and unit cells for (a) Si/Ge hetero-twinned SLs, (b) conventional Si/Ge SLs, (c) $\mathrm{Si}_{0.5} \mathrm{Ge}_{0.5}$ alloys, (d) single crystal $\mathrm{Si}$, and (e) twinned $\mathrm{Si}$ are shown. The blue atoms are $\mathrm{Si}$, and the green atoms are Ge.

In this work, molecular dynamic (MD) simulations ${ }^{19,20}$ have been applied to study the thermal transmission in $\mathrm{Si} / \mathrm{Ge}$ heterotwinned SLs by using LAMMPS package. ${ }^{21}$ In all MD simulations here, the Tersoff potential ${ }^{8}$ is used to describe the covalent interactions between $\mathrm{Si}$ and $\mathrm{Ge}$ atoms. Periodic boundaries are used in all three directions. Time step in all MD simulations are $1 \mathrm{fs}$. The atomic structures of Si/Ge hetero-twinned SLs are first equilibrated at a constant temperature of $T=300 \mathrm{~K}$ for $500 \mathrm{ps}$ using a NVT ensemble. After that, the non-equilibrium MD simulations (refer to ESI $\dagger$ ) are conducted on the relaxed structures for thermal conductivity calculation by using Fourier's law. ${ }^{22}$

\section{Results and discussion}

Thermal conductivities of $\mathrm{Si} / \mathrm{Ge}$ hetero-twinned SLs with different model lengths are calculated along [111], so as to eliminate the effect of model length on thermal conductivity of bulk Si/Ge hetero-twinned SLs. As suggested by Schelling et al. ${ }^{23}$ the inverse of thermal conductivity $(1 / K)$ is linearly related to the inverse of model length $(1 / L)$, which can be expressed by the following relationship:

$$
\frac{1}{K}=\frac{1}{K_{\infty}}\left(\frac{l_{\mathrm{SL}}}{L}+1\right)
$$

where $K_{\infty}$ is the thermal conductivity of bulk $\mathrm{Si} / \mathrm{Ge}$ heterotwinned SLs, and $l_{\mathrm{SL}}$ is the effective phonon mean free path in SLs. Thus, the thermal conductivity of bulk Si/Ge heterotwinned SLs can be obtained by extrapolating this linear relationship, as shown in Fig. S1. $\dagger$ In this way, thermal conductivities of bulk Si/Ge hetero-twinned SLs with different periodic thicknesses are calculated, and the results are plotted in Fig. 2. Thermal conductivities of bulk single crystal $\mathrm{Si}$, twinned $\mathrm{Si}$, $\mathrm{Si}_{0.5} \mathrm{Ge}_{0.5}$ alloy, and conventional $\mathrm{Si} / \mathrm{Ge}$ SLs along [111] are also calculated by NEMD simulation (refer to ESI $1 \dagger$ ) for comparison, and they are also shown in Fig. 2. Our simulated thermal conductivity of bulk single crystal Si is consistent with the previous reported results, ${ }^{24}$ demonstrating that our computational method is reliable. It can be noticed that when the periodic thickness $D_{\mathrm{P}}$ of Si/Ge hetero-twinned SLs varies from 1.93 to $57.83 \mathrm{~nm}$, their thermal conductivities are largely reduced compared with that of single $\mathrm{Si}$ crystal and $\mathrm{Si}_{0.5} \mathrm{Ge}_{0.5}$ alloy. In particular, when the periodic thickness is $15.4 \mathrm{~nm}$, the thermal conductivity of bulk Si/Ge hetero-twinned SLs can amount to only $17 \%$ of that of single $\mathrm{Si}$ crystal, and $36 \%$ of that of $\mathrm{Si}_{0.5} \mathrm{Ge}_{0.5}$ alloy. Moreover, comparison between thermal conductivities of bulk Si/Ge hetero-twinned SLs, twinned Si and conventional Si/ Ge SLs has also been made; it shows that when the periodic thickness of these three structures is the same, the thermal conductivities of bulk Si/Ge hetero-twinned and conventional $\mathrm{Si} / \mathrm{Ge}$ SLs are almost the same, while they are much smaller than that of bulk twinned Si. Thus, it can be concluded that the results show low thermal conductivity in bulk $\mathrm{Si} / \mathrm{Ge}$ heterotwinned SLs, and that phonon scattering in bulk Si/Ge heterotwinned SLs is much stronger than that in bulk single $\mathrm{Si}$ crystal, twinned $\mathrm{Si}$, and $\mathrm{Si}_{0.5} \mathrm{Ge}_{0.5}$ alloy, while it's almost same with that in the conventional $\mathrm{Si} / \mathrm{Ge}$ SLs.

It should also be noticed in Fig. 2 that thermal conductivity of bulk Si/Ge hetero-twinned SLs doesn't vary monotonously with the increase of periodic thickness, which has also been observed by many other researchers in superlattice systems., ${ }^{2,7,8}$ To explore the origin of this phenomenon, the total thermal

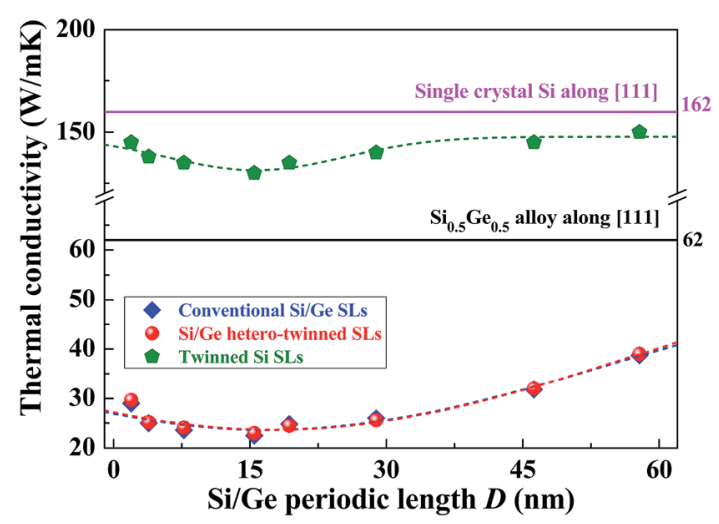

Fig. 2 Periodic thickness dependence of thermal conductivity for $\mathrm{Si} /$ Ge hetero-twinned SLs, in comparison with that of single crystal Si, twinned Si, Si/Ge alloy and conventional Si/Ge SLs. 
resistance of SLs $R_{\mathrm{SLS}}$ has been described by the summation of thermal resistance for individual layers as well as that for interfaces, as shown in eqn (2)

$$
R_{\mathrm{SLs}}=R_{\mathrm{Si}, \mathrm{N}}+R_{\mathrm{Ge}, \mathrm{N}}+R_{\text {interface }}
$$

where $R_{\mathrm{Si}, \mathrm{N}}$ is the total thermal resistance for individual $\mathrm{Si}$ layers, $R_{\mathrm{Ge}, \mathrm{N}}$ is the total thermal resistance for individual Ge layers, and $R_{\text {interface }}$ is the total thermal resistance of interfaces. For a SLs structure with a length of $L$, total length of Si and Ge layers are both $L / 2$, the number of $\mathrm{Si}$ and Ge layers are both $L / D$, and the number of interfaces is $2 L / D$, thus, eqn (2) leads to

$$
\frac{1}{K_{\mathrm{SLs}}}=\frac{1}{2 K_{\mathrm{Si}}}+\frac{1}{2 K_{\mathrm{Ge}}}+\frac{2 R_{\mathrm{K}}}{D}
$$

where $K_{\mathrm{SLs}}$ is the thermal conductivity of SLs, $K_{\mathrm{Si}}, K_{\mathrm{Ge}}$ are thermal conductivity of $\mathrm{Si}, \mathrm{Ge}$ individual layers. When the period thickness of individual layer $D$ is extremely larger than the phonon mean free path, phonon scattering within an individual layer will be diffusive, ${ }^{25}$ and thermal conductivity of individual Si and Ge layers equals to that of single crystal Si $K_{\mathrm{Si}-}$ sing and single crystal Ge $K_{\mathrm{Ge}-s i n g}$ (independent on the periodic thickness). As a result, eqn (3) takes the form

$$
\frac{1}{K_{\mathrm{SLs}}}=\frac{1}{2}\left(\frac{1}{K_{\mathrm{Si}-\text { sing }}}+\frac{1}{K_{\mathrm{Ge}-\text { sing }}}\right)+\frac{2 R_{\mathrm{K}}}{D}
$$

While when the periodic thickness is sufficiently smaller than the phonon mean free path, phonon scattering within the individual layer will be ballistically ${ }^{25}$ (no thermal resistance in individual layers), thus, the total thermal resistance of the superlattice equals to the total interface thermal resistance, and in this situation, eqn (3) can be expressed as

$$
\frac{1}{K_{\mathrm{SLs}}}=\frac{2 R_{\mathrm{K}}}{D}
$$

For periodic thickness comparable to phonon mean free path, combination of diffusive and ballistic phonon scattering will occur within the individual layer. By combining eqn (4) and (5), we can obtain that

$$
\frac{1}{K_{\mathrm{SLs}}}=M \times\left[\frac{1}{2}\left(\frac{1}{K_{\mathrm{Si}-\text { sing }}}+\frac{1}{K_{\mathrm{Ge}-\text { sing }}}\right)+\frac{2 R_{K}}{D}\right]+N \times \frac{2 R_{\mathrm{K}}}{D}
$$

where $M$ and $N$ are contribution of diffusive and ballistic phonon scattering in the thermal transmission of $\mathrm{Si} / \mathrm{Ge}$ heterotwinned SLs, and they satisfy that $M+N=1$. Moreover, eqn (6) can be used to express the thermal transmission in SLs for all possibilities with the variation of $M$ and $N$. Due to the linear relationship between the inverse of model length $1 / L$ and the inverse of thermal conductivity $1 / K_{\mathrm{Si} \text {-sing }}, 1 / K_{\mathrm{Ge}-\mathrm{sin}}, 1 / K_{\mathrm{SLS}}$ in eqn (6), it can be easily deduced that for a certain periodic thickness $D$, interface thermal resistance in bulk SLs $R_{\mathrm{K}-\mathrm{b}}$ is also linearly related to $1 / L$.

To obtain interface thermal resistance in bulk SLs $R_{\mathrm{K}-\mathrm{b}}$, NEMD simulations (refer to ESI $2 \dagger$ ) are carried out on $\mathrm{Si} / \mathrm{Ge}$ hetero-twinned SLs with different model length for different periodic thickness. Inset in Fig. 3 shows the model length dependent interface thermal resistance in $\mathrm{Si} / \mathrm{Ge}$ hetero-twinned SLs with $D=7.73 \mathrm{~nm}, 19.32 \mathrm{~nm}$ and $57.83 \mathrm{~nm}$, it can be seen that with the increase of periodic thickness, the interface thermal resistance is decreased, and the relationship between $R_{\mathrm{K}}$ and $1 / L$ is indeed linear, which can also be observed in other periodic thickness. By using extrapolation method, interface thermal resistance in the bulk SLs with different periodic thickness can be obtained, and results have been plotted in Fig. 3. It can be seen that with the periodic thickness decreases from $57.83 \mathrm{~nm}$ to $1.932 \mathrm{~nm}$, interface thermal resistance in the bulk SLs $R_{\mathrm{K}-\mathrm{b}}$ decreases from $5.34 \times 10^{-10} \mathrm{~m}^{2} \mathrm{~K} \mathrm{~W}^{-1}$ to $2.5 \times$ $10^{-11} \mathrm{~m}^{2} \mathrm{~K} \mathrm{~W}^{-1}$, especially when the periodic thickness decreases from $15.47 \mathrm{~nm}$ to $1.932 \mathrm{~nm}, R_{\mathrm{K}-\mathrm{b}}$ decreases rapidly and almost linearly. When the periodic thickness ranges from $1.932 \mathrm{~nm}$ to $15.47 \mathrm{~nm}$, coherent phonons exist. With the decrease of periodic thickness, the action of coherent becomes stronger. Such coherent phonon behavior will eliminate the effect of interface phonon scattering, ${ }^{26}$ thus, leading to rapid decrease of interface thermal resistance, and further causing the decrease of Si/Ge hetero-twinned SLs thermal conductivity with periodic thickness increases from $1.932 \mathrm{~nm}$ to $15.47 \mathrm{~nm}$. While when the periodic thickness ranges from $19.32 \mathrm{~nm}$ to $57.83 \mathrm{~nm}$, coherent phonons almost don't exist, and as a result, the interface thermal resistance becomes more and more close to a fixed value, and further, the thermal conductivity of $\mathrm{Si} / \mathrm{Ge}$ hetero-twinned SLs increases with increased periodic thickness. The nonmonotonic dependence of $\mathrm{Si} / \mathrm{Ge}$ hetero-twinned SLs on periodic thickness is caused by the combined action of phonon coherent and interface phonon scattering. ${ }^{26}$ Our results also support a hypothesis that in SLs with a short periodic thickness, there are almost no interfaces and the whole SLS should be considered as a giant lattice. ${ }^{27}$

To explore the strong phonon scattering in Si/Ge heterotwinned SLs, phonon density of states (PDOS) for $\mathrm{Si} / \mathrm{Ge}$ hetero-twinned SLs with a periodic thickness of $1.93 \mathrm{~nm}$, as

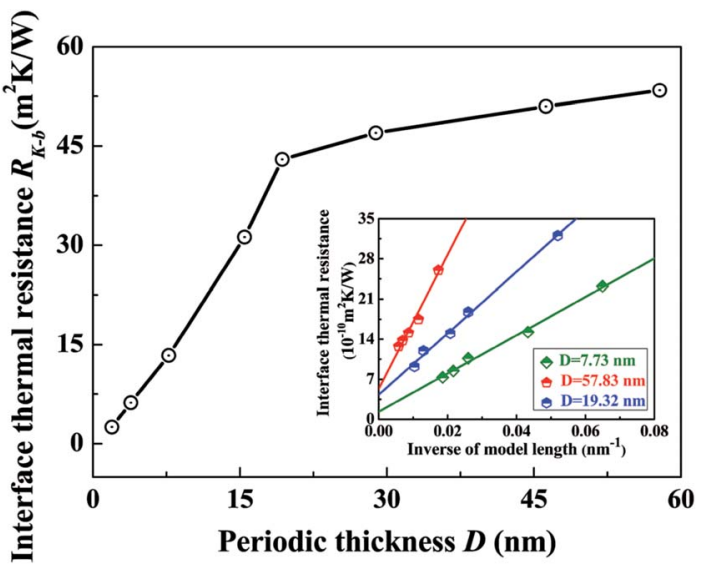

Fig. 3 Periodic thickness dependent interface thermal resistance in bulk Si/Ge hetero-twinned SLs. Inset: linear relationship between the inverse of model length and interface thermal resistance for $\mathrm{Si} / \mathrm{Ge}$ hetero-twinned SLs with a periodic thickness of $D=7.73 \mathrm{~nm}, 19.32 \mathrm{~nm}$ and $57.83 \mathrm{~nm}$ 
well PDOS that for single crystal $\mathrm{Si}$, twinned $\mathrm{Si}, \mathrm{Si}_{0.5} \mathrm{Ge}_{0.5}$ alloy, and conventional $\mathrm{Si} / \mathrm{Ge} \mathrm{SLs}$ with similar structures and the same atom number, are calculated by using PHONOPY. ${ }^{28}$ As can be seen from Fig. 4, the PDOS of Si/Ge hetero-twinned SLs is similar to that of the conventional Si/Ge SLs, and the high frequency peaks in the PDOS of the two structures are both approximately at $8.1 \mathrm{THz}$, thus resulting in the similar thermal conductivity for them. At the same time, when compared with single crystal $\mathrm{Si}$, twinned $\mathrm{Si}$ and $\mathrm{Si}_{0.5} \mathrm{Ge}_{0.5}$ alloy, there is a distinct redshift for the PDOS in Si/Ge hetero-twinned SLs, which indicates the possibility of increased phonon scattering in $\mathrm{Si} / \mathrm{Ge}$ hetero-twinned SLs, under the condition that the total number of phonon modes is exactly the same for the above five structures. Thus, it can be concluded that the redshift of the phonons with high frequency results in the low thermal conductivity of $\mathrm{Si} / \mathrm{Ge}$ hetero-twinned SLs. ${ }^{29}$ Besides, the similar PDOS of bulk twinned Si and the single crystal Si can also be observed, which indicates the weak phonon scattering at twin boundaries.

Since it is well known that phonons are primary heat carriers in semiconductors, ${ }^{30}$ low thermal conductivity of bulk $\mathrm{Si} / \mathrm{Ge}$ hetero-twinned SLs has also been studied by phonon kinetic theory, in which thermal conductivity $K$ can be described by ${ }^{31}$

$$
K=\frac{1}{3} C_{\mathrm{V}} v_{\mathrm{g}} l=\frac{1}{3} C_{\mathrm{V}} v_{\mathrm{g}}{ }^{2} \tau,
$$

where $C_{\mathrm{V}}$ is the specific heat capacity, $v_{\mathrm{g}}$ is the average phonon group velocity, $l$ is the phonon mean free path, and $\tau$ is the relaxation time.

In comparison with thermal transmission in bulk $\mathrm{Si} / \mathrm{Ge}$ hetero-twinned SLs $\left(D_{\mathrm{P}}=1.93 \mathrm{~nm}\right)$, this characteristic has also been analyzed for single crystal $\mathrm{Si}$, twinned $\mathrm{Si}, \mathrm{Si}_{0.5} \mathrm{Ge}_{0.5}$ alloy and conventional $\mathrm{Si} / \mathrm{Ge}$ SLs with similar structures and the same atom number by phonon kinetic theory. Specific heat capacities of these structures at room temperature are calculated by using PHONOPY firstly (details can be found in ESI $3 \dagger$ ), and results are summarized in Fig. 5(a). It can be seen that with the increase in temperature, the specific heat capacity is increased, and finally it reaches a constant value when the

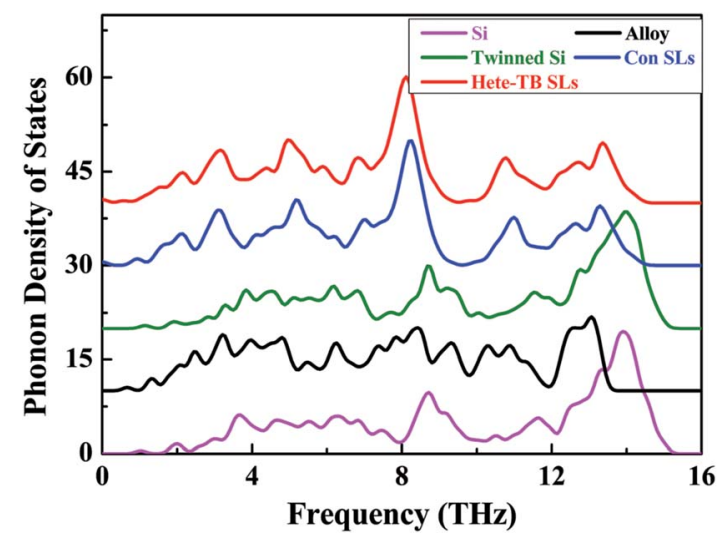

Fig. 4 Comparison of phonon density of states for Si/Ge heterotwinned SLs (the red), single crystal Si (the green), twinned Si (the pink), $\mathrm{Si}_{0.5} \mathrm{Ge}_{0.5}$ alloy (the black) and conventional Si/Ge SLs (the blue). temperature is between 800 and $1000 \mathrm{~K}$. For single crystal Si and twinned $\mathrm{Si}$, the value $\mathrm{e}^{32}$ is $2.1 \times 10^{6} \mathrm{~J} \mathrm{~K}^{-1} \mathrm{~m}^{3}$, and for bulk Si/Ge hetero-twinned $\mathrm{SLs}, \mathrm{Si}_{0.5} \mathrm{Ge}_{0.5}$ alloy and conventional $\mathrm{Si} / \mathrm{Ge} \mathrm{SLs}$, the value is $1.95 \times 10^{6} \mathrm{~J} \mathrm{~K}^{-1} \mathrm{~m}^{3}$. A crossover of specific heat capacities for the five structures occurs at $300 \mathrm{~K}$, and this distinct value ${ }^{32}$ is $1.71 \times 10^{6} \mathrm{~J} \mathrm{~K}^{-1} \mathrm{~m}^{3}$. Thus, it can be concluded that when compared with the other four structures, low thermal conductivity in Si/Ge hetero-twinned SLs is not related to the specific heat capacity at room temperature.
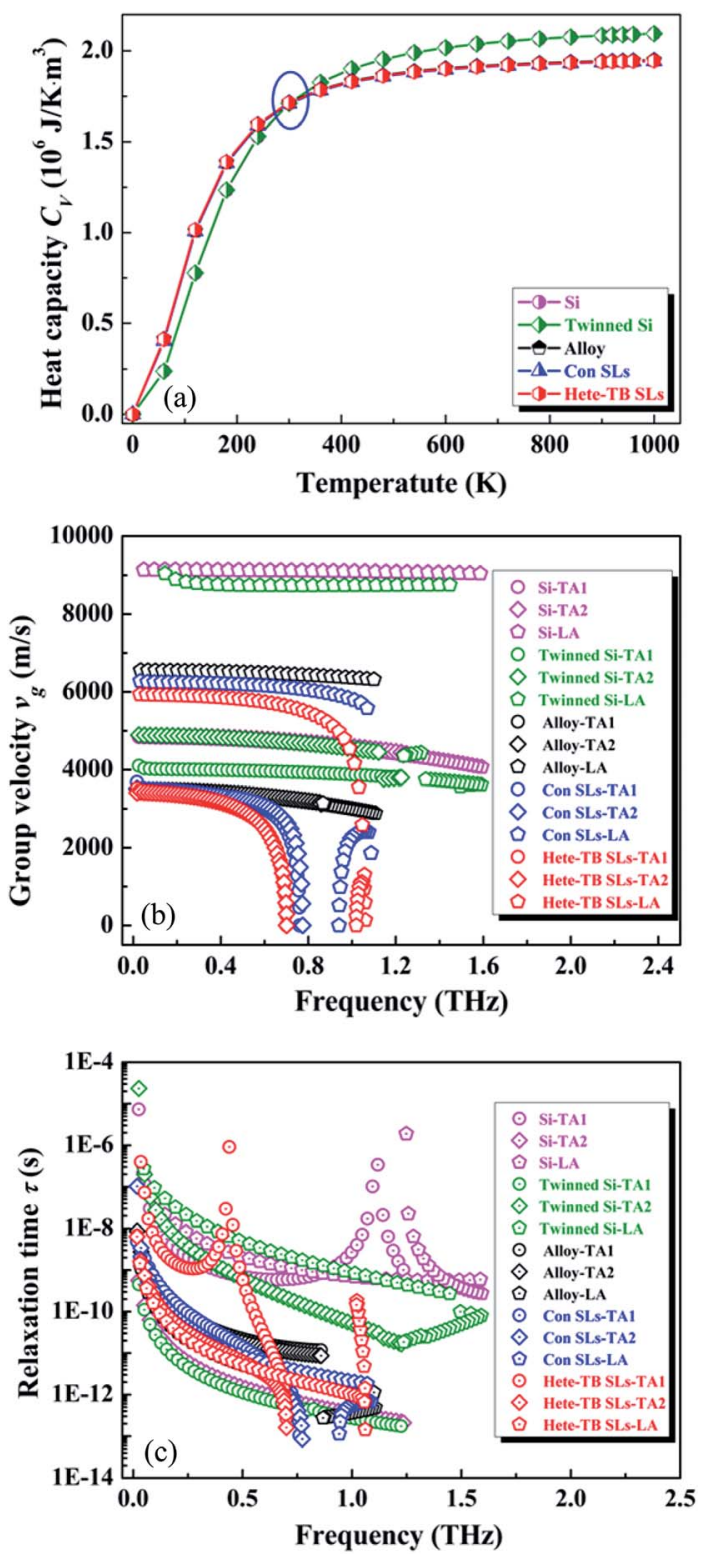

Fig. 5 Comparison of (a) specific heat capacity, (b) phonon group velocity, and (c) relaxation time for $\mathrm{Si} / \mathrm{Ge}$ hetero-twinned SLs, single crystal Si, twinned $\mathrm{Si}, \mathrm{Si}_{0.5} \mathrm{Ge}_{0.5}$ alloy and conventional Si/Ge SLs. For (b) and (c), only the acoustic branches are shown, "TA1", "TA2" and "LA" correspond to two transverse branches and one longitudinal branch. "Si" corresponds to single Si crystal, "Twinned $\mathrm{Si}^{\prime}$ corresponds to twinned Si crystal, "Alloy" corresponds to $\mathrm{Si}_{0.5} \mathrm{Ge}_{0.5}$ alloy, "Con-SLs" corresponds to conventional Si/Ge SLs, and "Hete-TB SLs" corresponds to Si/Ge hetero-twinned SLs. 
The phonon group velocity can be obtained from the following relationship ${ }^{33}$

$$
v_{\mathrm{g}}=(\partial \omega / \partial q)
$$

where $q$ is the wave vector, and $\omega$ is the frequencies of both the acoustic and optical branches in phonon spectra. Fig. S3† presents phonon dispersion of acoustic branches (longitudinal and two degenerated transverse modes), which are the primary carriers of thermal transmission, for the five structures. It is clearly shown that compared with bulk single crystal Si, twinned $\mathrm{Si}$ and $\mathrm{Si}_{0.5} \mathrm{Ge}_{0.5}$ alloy, dispersion curves of $\mathrm{Si} / \mathrm{Ge}$ hetero-twinned SLs are dramatically depressed, and moreover, they are also gentler than that of the conventional Si/Ge SLs. By applying eqn (8), phonon group velocity $v_{\mathrm{g}}$ for the five structures at different frequencies is calculated. As shown in Fig. 5(b), $v_{\mathrm{g}}$ of $\mathrm{Si} / \mathrm{Ge}$ hetero-twinned SLs at each frequency is smaller than that of the four other structures. As a result, the average value of $v_{\mathrm{g}}$ for $\mathrm{Si} / \mathrm{Ge}$ hetero-twinned SLs $\left(3273 \mathrm{~m} \mathrm{~s}^{-1}\right)$ is lower than that of conventional Si/Ge SLs (3619 $\mathrm{m} \mathrm{s}^{-1}$ ), Si/Ge alloy (3999 $\mathrm{m} \mathrm{s}^{-1}$ ), single Si crystal (5078 $\mathrm{m} \mathrm{s}^{-1}$ ) and twinned Si $\left(5615 \mathrm{~m} \mathrm{~s}^{-1}\right)$, as shown in Table 1. Thus, the reduced phonon group velocity in $\mathrm{Si} / \mathrm{Ge}$ hetero-twinned SLs largely contributes to the low thermal conductivity in Si/Ge hetero-twinned SLs according to eqn (7). It's worth noting the lower phonon group velocity in $\mathrm{Si} / \mathrm{Ge}$ hetero-twinned SLs compared to that of the conventional $\mathrm{Si}$ / Ge SLs; this indicates that despite of the similar thermal conductivities in the two structures, they show different effects on phonon group velocity.

In addition to the volume heat capacity and phonon group velocity, relaxation time $\tau$ is also a significant factor that affects thermal transmission in $\mathrm{Si} / \mathrm{Ge}$ hetero-twinned SLs. The relaxation time $\tau$ can be obtained by applying a semi-empirical theory, in which $\tau$ strongly depends on the anharmonicity of the bonding, and it can be expressed by ${ }^{\mathbf{3 4 , 3 5}}$

$$
\tau(\omega)=A \frac{\bar{M} v_{\mathrm{g}} v_{\mathrm{p}}^{2}}{k_{\mathrm{B}} a \gamma^{2} \omega^{2} T},
$$

where $A$ is a parameter related to the intrinsic material properties, $M$ is the average mass of the cell, $v_{\mathrm{g}}$ and $v_{\mathrm{p}}$ are the group and phase velocities respectively, $k_{\mathrm{B}}$ is Boltzmann constant, $a^{3}$ is the volume per atom, $\omega$ is phonon frequency, $T$ is temperature,

Table 1 Calculated average values of group velocity $\left(\overline{v_{g}}\right)$, phase velocity $\left(\overline{v_{p}}\right)$, Grüneisen parameter $(\bar{\gamma})$, relaxation time $(\bar{\tau})$, thermal conductivity from phonon kinetic theory $\left(K_{\mathrm{PH}}\right)$ and in MD simulation $\left(K_{\mathrm{MD}}\right)$ for Si/Ge hetero-twinned SLs (Hete-TB SLs), single crystal Si (Si), twinned $\mathrm{Si}, \mathrm{Si}_{0.5} \mathrm{Ge}_{0.5}$ alloy (Alloy) and conventional Si/Ge SLs (Con SLs)

\begin{tabular}{llllll}
\hline & $\begin{array}{l}\text { Hete-TB } \\
\text { SLs }\end{array}$ & $\begin{array}{l}\text { Con } \\
\text { SLs }\end{array}$ & Si & $\begin{array}{l}\text { Twinned } \\
\text { Si }\end{array}$ & Alloy \\
\hline$\overline{v_{\mathrm{g}}}\left(\mathrm{m} \mathrm{s}^{-1}\right)$ & 3273 & 3619 & 5615 & 5078 & 3999 \\
$\overline{v_{\mathrm{p}}}\left(\mathrm{m} \mathrm{s}^{-1}\right)$ & 3997 & 4140 & 5948 & 5943 & 4211 \\
$\bar{\gamma}$ & 0.447 & 0.632 & 0.407 & 0.387 & 0.564 \\
$\bar{\tau}(\mathrm{ps})$ & 5.11 & 4.21 & 9.03 & 8.97 & 5.42 \\
$K_{\mathrm{PH}}\left(\mathrm{W} \mathrm{mK}^{-1}\right)$ & 31.2 & 31.4 & 162.3 & 131.8 & 49.4 \\
$K_{\mathrm{MD}}\left(\mathrm{W} \mathrm{mK}^{-1}\right)$ & 28.5 & 29 & 162 & 138 & 62
\end{tabular}

and $\gamma$ is the mode Grüneisen parameter, ${ }^{36}$ which, for each mode $i$ and frequency $\omega$, is defined as the logarithmic derivative of the phonon frequency $\omega$ with respect to the volume $V$ of the crystal that $\gamma_{\mathrm{i}}(\omega) \equiv d \ln \omega /\left.d \ln V\right|_{\mathrm{j}}$.

To calculate the relaxation time of $\mathrm{Si} / \mathrm{Ge}$ hetero-twinned SLs, both frequency-dependent phase velocities and Grüneisen parameters have been calculated. The same has been done for bulk single crystal $\mathrm{Si}$, twinned $\mathrm{Si}$ and $\mathrm{Si}_{0.5} \mathrm{Ge}_{0.5}$ alloy and conventional Si/Ge SLs. As shown in Fig. S5, $\uparrow$ when compared with single Si crystal, the existence of twin boundaries almost has no effect on phase velocity, while the phase velocity of $\mathrm{Si} / \mathrm{Ge}$ hetero-twinned SLs is largely reduced, and it is even lower than that of conventional Si/Ge SLs. Consequently, the average phase velocity of $\mathrm{Si} / \mathrm{Ge}$ hetero-twinned SLs is the lowest among the five structures, as shown in Table 1. As for Grüneisen parameters, which have been plotted in Fig. S6, $\uparrow$ compared with the other four structures, the existence of $\mathrm{Si} / \mathrm{Ge}$ hetero-twinned boundaries has strong effect on it at different frequencies. The average values of Grüneisen parameters for the five structures can also be found in Table 1. It can be seen that compared with single crystal Si, Grüneisen parameters of Si/Ge hetero-twinned SLs, the conventional $\mathrm{Si} / \mathrm{Ge} \mathrm{SLs}$ and $\mathrm{Si} / \mathrm{Ge}$ alloy are increased, while this parameter for the twinned Si is only slightly reduced. After calculation of phase velocity and Grüneisen parameters, according to eqn (9), $A$ is the only parameter needed to be determined. To this end, the average values of group velocity, phase velocity and Grüneisen parameters of single crystal Si in Table 1 are used, and the value of $A$ is determined as $9.1 \times 10^{-5}$. Assuming that for all the five structures, value of $A$ is the same, thus eqn (9) leads to

$$
\tau(\omega)=9.1 \times 10^{-5} \frac{\bar{M} v_{\mathrm{g}} v_{\mathrm{p}}{ }^{2}}{k_{\mathrm{B}} a \gamma^{2} \omega^{2} T},
$$

Fig. 5(c) shows the frequency-dependent relaxation time $\tau$ for the five structures. It can be seen that relaxation time differs largely with the variation of phonon frequency. Especially, when the frequency is low, the relaxation time can amount to more than $10 \mathrm{~ns}$, which is consistent with the previous results for LA branch in single crystal $\mathrm{Si}^{37}$ For one branch of acoustic transverse modes in twinned $\mathrm{Si}, \tau_{\mathrm{Si} \text {-TB }}$, is almost the same with that of the single Si crystal $\tau_{\mathrm{Si}}$, while $\tau_{\mathrm{Si} \text {-тв }}$ differs from $\tau_{\mathrm{Si}}$ for the left branch of acoustic transverse mode and longitudinal mode; $\tau_{\mathrm{Si}-}$ тв is lower than $\tau_{\mathrm{Si}}$, especially at the frequency between 1 to 1.3 THz. The Moreover, different relaxation times of $\mathrm{Si} / \mathrm{Ge}$ heterotwinned $\tau_{\mathrm{Si} / \mathrm{Ge}-\mathrm{TB}}$ and conventional $\mathrm{Si} / \mathrm{Ge}$ SLs $\tau_{\mathrm{Si} / \mathrm{Ge}}$ can also be observed. For one acoustic transverse branch, $\tau_{\mathrm{Si} / \mathrm{Ge}-\mathrm{TB}}$ is higher than $\tau_{\mathrm{Si} / \mathrm{Ge}}$ at the frequency between 0 to about $0.5 \mathrm{THz}$. The same is true for the acoustic longitudinal branch at the frequency close to $1 \mathrm{THz}$. Considering the combined actions of phonons in the entire frequency range, the integrated relaxation time was calculated by inserting average values of parameters in eqn (10). As shown in Table 1, when compared with single Si crystal, the relaxation time of twinned $\mathrm{Si}$ is only slightly reduced, indicating the weak effect of twin boundaries on relaxation time. The relaxation time of $\mathrm{Si} / \mathrm{Ge}$ hetero-twinned SLS is largely reduced, and it makes a significant contribution to the 
low thermal conductivity in Si/Ge hetero-twinned SLs. Moreover, it can also be observed that the relaxation time of $\mathrm{Si} / \mathrm{Ge}$ hetero-twinned SLs is larger than that of conventional $\mathrm{Si} / \mathrm{Ge}$ SLs, which demonstrates the different effects of the two structures on relaxation time.

According to eqn (7), the thermal conductivity can be obtained by a relationship connecting the heat capacity, group velocity, and relaxation time. Calculated results for the five structures by phonon kinetic theory have also been presented in Table 1. It can be seen that compared with single crystal Si, twinned $\mathrm{Si}$ and $\mathrm{Si}_{0.5} \mathrm{Ge}_{0.5}$ alloy, thermal conductivity of $\mathrm{Si} / \mathrm{Ge}$ hetero-twinned SLs is much lower, while it's close to that of conventional $\mathrm{Si} / \mathrm{Ge} \mathrm{SLs}$, which is consistent with the results obtained by NEMD simulations. By combining the calculated group velocity and relaxation time in Table 1 , it can be deduced that the low thermal conductivity in Si/Ge hetero-twinned SLs is caused by the combined actions of reduced phonon group velocity and reduced relaxation time. Furthermore, it can also be observed that despite of similar thermal conductivities in $\mathrm{Si}$ / Ge hetero-twinned SLs and conventional Si/Ge SLs, the average phonon group velocity in Si/Ge hetero-twinned SLs is lower, while the relaxation time in it is larger, which makes up the smaller group velocity.

\section{Conclusion}

In conclusion, by employing nonequilibrium molecular dynamic simulations, thermal transport in $\mathrm{Si} / \mathrm{Ge}$ heterotwinned SLs have been studied. The results indicate that compared with single crystal $\mathrm{Si}$, twinned $\mathrm{Si}$ and $\mathrm{Si}_{0.5} \mathrm{Ge}_{0.5}$ alloy, $\mathrm{Si} / \mathrm{Ge}$ hetero-twinned SLs show low thermal conductivity, which is similar to that of conventional Si/Ge SLs. Phonon kinetic theory has also been applied to analyze the low thermal conductivity, and results show that low thermal conductivity in $\mathrm{Si} / \mathrm{Ge}$ hetero-twinned SLs is caused by the combined actions of reduced phonon group velocity and reduced relaxation time. Moreover, despite of similar thermal conductivities in $\mathrm{Si} / \mathrm{Ge}$ hetero-twinned SLs and conventional Si/Ge SLs, which is caused by the combined action of umklapp phonon scattering and interface scattering, phonon group velocity and relaxation time in the two structures are fundamentally different that the average phonon group velocity in Si/Ge hetero-twinned SLs is lower, while the larger average relaxation time in $\mathrm{Si} / \mathrm{Ge}$ heterotwinned SLs makes up the lower group velocity exactly. Our results demonstrate a new kind of SLs with strong phonon scattering.

\section{Acknowledgements}

This work was supported by the National Natural Science Foundation of China under grant numbers 51372215. Y. Z. acknowledges the support from the U.S. National Science Foundation under grant number CBET-1404482. R. M. acknowledges the support from the NSERC and CRC programs, Canada.

\section{References}

1 Y. Chen, D. Li, J. Yang, Y. Wu, J. R. Lukes and A. Majumdar, Phys. B, 2004, 349, 270-280.

2 Y. Chen, D. Li, J. R. Lukes, Z. Ni and M. Chen, Phys. Rev. B: Condens. Matter Mater. Phys., 2005, 72, 174302.

3 Y. K. Koh, Y. Cao, D. G. Cahill and D. Jena, Adv. Funct. Mater., 2009, 19, 610-615.

4 Y. Chalopin, K. Esfarjani, A. Henry, S. Volz and G. Chen, Phys. Rev. B: Condens. Matter Mater. Phys., 2012, 85, 195302.

5 Z. Tian, K. Esfarjani and G. Chen, Phys. Rev. B: Condens. Matter Mater. Phys., 2012, 86, 235304.

6 V. Samvedi and V. Tomar, Nanotechnology, 2009, 20, 365701.

7 J. Garg and G. Chen, Phys. Rev. B: Condens. Matter Mater. Phys., 2013, 87, 140302.

8 M. Hu and D. Poulikakos, Nano Lett., 2012, 12, 5487-5494.

9 A. Porter, C. Tran and F. Sansoz, Phys. Rev. B, 2016, 93, 195431.

10 M. I. H. E. S. Landry and A. J. H. McGaughey, Phys. Rev. B: Condens. Matter Mater. Phys., 2008, 77, 184302.

11 T. Borca-Tasciuc, W. Liu, J. Liu, T. Zeng, D. W. Song, C. D. Moore, G. Chen, K. L. Wang, M. S. Goorsky, T. Radetic, R. Gronsky, T. Koga and M. S. Dresselhaus, Superlattices Microstruct., 2000, 28, 199-206.

12 S. Xiong, Y. A. Kosevich, K. Sääskilahti, Y. Ni and S. Volz, Phys. Rev. B: Condens. Matter Mater. Phys., 2014, 90, 195439.

13 S. M. Lee, D. G. Cahill and R. Venkatasubramanian, Appl. Phys. Lett., 1997, 70, 2957.

14 Y. T. Shin-ichiro Tamura, Phys. Rev. B: Condens. Matter Mater. Phys., 1999, 60, 2627-2630.

15 H. Dong, J. Xiao, R. Melnik and B. Wen, Sci. Rep., 2016, 6, 19575.

16 Y. Liu, D. Bufford, H. Wang, et al., Acta Mater., 2011, 59, 1924.

17 J. Li, Y. Chen, S. Xue, et al., Acta Mater., 2016, 114, 154.

18 T. Fu, X. Peng, X. Chen, S. Weng, N. Hu, Q. Li and Z. Wang, Sci. Rep., 2016, 6, 35665.

19 D. R. F. Müller-Plathe, Comput. Theor. Polym. Sci., 1999, 9, 203-209.

20 F. Müller-Plathe, J. Chem. Phys., 1997, 106, 6082.

21 S. Plimpton, J. Chem. Phys., 1995, 117, 1-19.

22 A. Bagri, S.-P. Kim, R. S. Ruoff and V. B. Shenoy, Nano Lett., 2011, 11, 3917-3921.

23 P. K. Schelling, S. R. Phillpot and P. Keblinski, Phys. Rev. B: Condens. Matter Mater. Phys., 2002, 65, 144306.

24 H. R. Shanks, P. D. Maycock, P. H. Sidles, et al., Phys. Rev., 1963, 130, 1743.

25 B. Saha, Y. R. Koh, J. Comparan, S. Sadasivam, J. L. Schroeder, M. Garbrecht, A. Mohammed, J. Birch, T. Fisher, A. Shakouri and T. D. Sands, Phys. Rev. B, 2016, 93, 045311.

26 M. Hu and D. Poulikakos, Nano Lett., 2012, 12, 5487.

27 M. Kazan and P. Masri, Surf. Sci. Rep., 2014, 69, 1-37.

28 G. Kresse and J. Hafner, Phys. Rev. B: Condens. Matter Mater. Phys., 1993, 47, 558-561. 
29 Y. Gao, X. Zhang, Y. Jing and M. Hu, Nanoscale, 2015, 7, 7143-7150.

30 J. Zou and A. Balandin, J. Appl. Phys., 2001, 89, 2932-2938.

31 Z. Wang, J. E. Alaniz, W. Jang, J. E. Garay and C. Dames, Nano Lett., 2011, 11, 2206-2213.

32 M. Maldovan, J. Appl. Phys., 2011, 110, 114310.

33 X. Feng, J. Xiao, R. Melnik, Y. Kawazoe and B. Wen, J. Chem. Phys., 2015, 143, 104503.
34 Y. Ding, B. Xiao, G. Tang and J. Hong, J. Phys. Chem. C, 2016, 121, 225-236.

35 E. S. Toberer, A. Zevalkink and G. J. Snyder, J. Mater. Chem., 2011, 21, 15843.

36 H. Jin, O. D. Restrepo, N. Antolin, S. R. Boona, W. Windl, R. C. Myers and J. P. Heremans, Nat. Mater., 2015, 14, 601606.

37 A. Ward and D. A. Broido, Phys. Rev. B: Condens. Matter Mater. Phys., 2010, 81, 085205. 